BNL- 64800

UC-414

AGS/AD/98-1

INFORMAL

\title{
A STUDY OF LIGHT ION ACCELERATORS FOR CANCER TREATMENT
}

K. Prelec

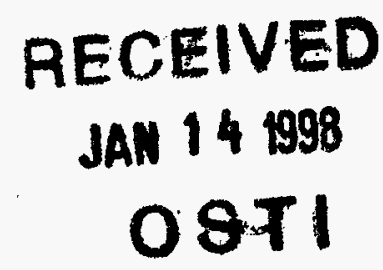

October 1, 1997

DISTRIBUTION RESTRICTED TO U.S. ONLY ph

ALTERNATING GRADIENT SYNCHROTRON DEPARTMENT

BROOKHAVEN NATIONAL LABORATORY

ASSOCIATED UNIVERSITIES, INC.

UPTON, LONG ISLAND, NEW YORK 


\section{DISCLAIMER}

This report was prepared as an account of work sponsored by the United States Government Neither the United States nor the United States Deparment of Energy, nor any of their employees, nor any of their contractors, subcontractors, or their employees, makes any warranty, express or implied, or assumes any legal liability or responsibility for the accuracy, completeness, or usefulness of any information, apparatus, product or process disclosed, or represents that its use would not infringe privately owned rights. 


\section{DISCLAMnixR}

Portions of this document may be illegible in electronic image produets. Images are produced from the best available original docoment 


\section{INTRODUCTION}

Radiation therapy has become one of the most important modalities in the treatment of cancer. It is estimated that a person has one out of three chance to be confronted with the disease during his or her lifetime and that less than half of them will be cured. While the surgery is still the most successful treatment, radiation therapy either alone or in combination with other modalities contributes to about $40 \%$ of the overall cure rates. It is interesting to note that chemotherapy alone results in a rather small part of cancer cures; it is used mostly as an adjuvant therapy. All other modalities contribute only a few percent to the cure rates.

Ideally, the objective of any cancer treatment method is to remove or destroy the tumor while preserving at the same time the healthy tissue as much as possible. It was with this idea in mind that almost a hundred years ago low energy $\mathrm{X}$-rays began to be used for this purpose, although their penetration was poor and therapeutic effect debatable. In early 1920's radium units came into use, producing deeper penetrating gamma rays; this was followed by electron accelerators providing higher energy X-rays. Nuclear reactors made radioactive cobalt sources available and they became a standard gamma ray source for radiotherapy, used until the present (e.g., gamma knife). Most modern and very widely used machines for $\mathrm{X}$-ray therapy are compact linear accelerators and it is estimated that there are up to 4000 of them around the world. Over the years this technique has been constantly improved, the machines have been adapted to the hospital environment and the delivery of the radiation to the tumor has become more and more accurate, trying at the same time to spare the healthy tissues. However, there are still many cases where it is not possible to avoid irradiation of critical organs in the vicinity of the tumor; the maximum dose allowed for critical organs would in such cases limit the dose given to the tumor, leading to a possible failure of the local control.

About fifty years ago $\mathrm{R}$. Wilson remarked that the Bragg peak of monoenergetic protons (and other, heavier ions) would allow the radiation dose to be preferentially delivered at the end of their path, in the tumor itself where most damage has to be done. By modulating the proton (or ion) energy it would in principle be possible to irradiate the whole volume of the tumor with a uniform and sufficient dose, while keeping the dose delivered to other organs at a lower value. This characteristic together with a high lateral beam accuracy is the basis of conformal treatment of tumors, which is an important step toward the ideal method. Since this, first, proposal there were a number of proton machines either adapted or specifically built for tumor treatment and proton beams have established their place in the radiotherapy.

The most recent and quite promising introduction into the range of types of radiation for cancer treatment have been energetic ions in the mass range from carbon to neon. They will be referred to as light ions which is the accepted use in physics, although in the medical literature they are usually called heavy ions. In addition to the advantage of showing a Bragg peak which is a similar characteristic of protons, and an even better lateral beam accuracy than protons, ions have other characteristics which could make them more suitable for treatment of some types of tumors than any other radiation. The Linear Energy Transfer (LET) or the rate of energy deposition along the path of a particle is higher for light ions (fast neutrons have a similar property) than it is for conventional radiation, including protons; the Relative Biological Effectiveness (RBE) tends to be higher if LET values are higher. Treatment of cancer fails very often because of the failure of the local tumor control; in some cases this may be due to the fact that in some tumors cells are anoxic and as such are more resistant to conventional radiation. The oxygen effect is characterized by the 
Oxygen Enhancement Ratio (DER) and for conventional radiation this factor may be as high as 3, meaning that it would take thee times higher dose of a particular kind of radiation to achieve the same effect in the tumor as in healthy tissues. On the other hand, for light ions the oxygen effect may be very close to 1 . There are also indications that the effects of light ion radiation in the tumor do not depend as much on the cell cycle as they do for conventional radiation.

However, possible advantages of light ions compared to conventional radiation result in a more complex system for beam production and, especially, for beam delivery to the patient.With high LET particles and with a large part of their energy delivered at the end of the track, in the Bragg peak, it becomes extremely imfiortant to properly adjust not only the shape of the beam but its energy and the time spent irradiating a certain part of the tumor as well; otherwise, healthy tissues may be exposed unnecessarily while the tumor may not get the required dose. The proper utilization of light ions requires a state-of-art application of medical diagnostics (CT, MRI, PET) to determine the exact shape and location of the tumor, a full computer control of the accelerator and beam delivery system and a fast and accurate measurement of the beam dose delivered at any instant to the patient. This complexity of the system has until very recently been one of the reasons that light ions have found a very limited use in medicine, particularly in the treatment of cancer so that at present there is just one dedicated facility in the operation (Chiba, Japan). Another reason for the lack of interest was the fact that in the past the few accelerators capable of producing light ion beams with parameters adequate for medical applications were designed for a totally different purpose (nuclear and particle physics), with ene:gies and intensities not matched to the needs of patient treatment, complicated to use and expensive to operate. Compared to light ion accelerators, electron linacs for photon production have enjoyed a long history of development and present designs are well adapted to a hospital environment.

This review will try to acidress several issues, such as possible advantages of light ion therapy compared to protons and conventional radiation, the complexity of such a system and its possible adaptation to a hospital envircinment, and the question of cost-effectiveness compared to other modalities for cancer treatment or to other life saving procedures.Characteristics and effects of different types of radiation on cells and organisms will be briefly described; this will include conventional radiation, protons and light ions. The status of proton and light ion cancer therapy will then be described, with more emphasis on the latter; on the basis of existing experience the criteria for the use of light ions will be listed and areas of possible medical applications suggested. Requirements and parameters of ion beams for cancer treatment will then be defined, including ion species, energy and intensity, as well as parameters of the beam when delivered to the target (scanning, time structure, ene:rgy spread). Possible accelerator designs for light ions will be considered, including linear accelerators, cyclotrons and synchrotrons and their basic features given; this will be followed by a review of existing and planned facilities for light ions. On the basis of these considerations a tentative design for a dedicated light ion facility will be suggested, a facility that would be hospital based, satisfying the clinical requirements, simple to operate and reliable, concluding with its cost-effectiveness in comparison with other modalities for treatment of cancer. 


\section{CHARACTERISTICS AND EFFECTS OF RADIATION}

The objective of any cancer treatment is the control or, possibly, permanent elimination of the tumor. As this process involves and results in cell destruction, the success of the treatment will always depend on the degree of discrimination between healthy tissues and tumor itself. From its early days about a hundred years ago radiation was recognized as having properties that could destroy the tumor; the problem, however, has always been how to deliver the required dose to the target without damaging beyond repair the surrounding healthy tissues including possibly critical organs. The improvements and development of new ways to deliver the radiation to the tumor or to explore new (for such an application) types of radiation have always been prompted by the desire to concentrate as much of the dose as possible to the tumor because it is the tolerance of adjacent organs that will limit the tumor dose. It follows also that if the damage to adjacent organs caused by radiation determines and limits the maximum dose that can be delivered to the tumor, the result could be a failure of the local control and failure of the treatment itself.

Conventional types of radiation, which include gamma-rays from radioactive isotopes, brehmsstrahlung photons and electrons have been used widely and routinely for treatment of human cancer since the earliest days (the word "conventional" is used because of historical reasons, without implying limitations or a lower quality). It is a common property of conventional radiation that the dose delivered to the body is the highest in the entrance region and decreases as the radiation penetrates the body (Fig.1). For gamma-rays and photons the decrease has an exponential character, which means that after passing through the target the radiation dose decreases further but healthy tissues and possibly critical organs beyond the tumor are still being irradiated; the effect of radiation on healthy tissues both in front and behind the tumor may therefore limit the dose delivered to the tumor. The photon energy is transferred to the tissues through stochastic processes, such as inelastic scattering or photoelectric processes, resulting in another effect that has to be taken into consideration: a photon beam is subject to strong scattering as it penetrates the body and this leads to a lateral beam spreading. Photons and gamma-rays are called indirectly ionizing because the biological effect is due to the action of fast electrons produced in tissues. High energy electrons are directly ionizing particles; the dose delivered is decreasing even faster with depth than for photons but they have a finite range if the energy is properly selected (Fig.1). They also show the effects of a strong scattering, resulting in a lateral spreading of the beam as it penetrates the body. The delivery of conventional radiation can be substantially improved if the tumor is irradiated from several directions, by rotating the beam around the patient and aiming it toward the isocenter. Although this complicates the beam delivery system, the result is a more favorable ratio of the doses in the tumor and outside. In the case of gamma rays from the radioactive cobalt, application of this method has led to the creation of the so called "gamma knife" where the rays from more than a hundred sources are collimated and directed toward the tumor enabling its destruction. Compared to other types of radiation, the conventional radiation is at present by far the most widely used type, contributing almost exclusively to a substantial part of cancer cure rates. Their most efficient use is in the case of fast growing tumors because those cells divide frequently and photons act especially on cells during their division. 


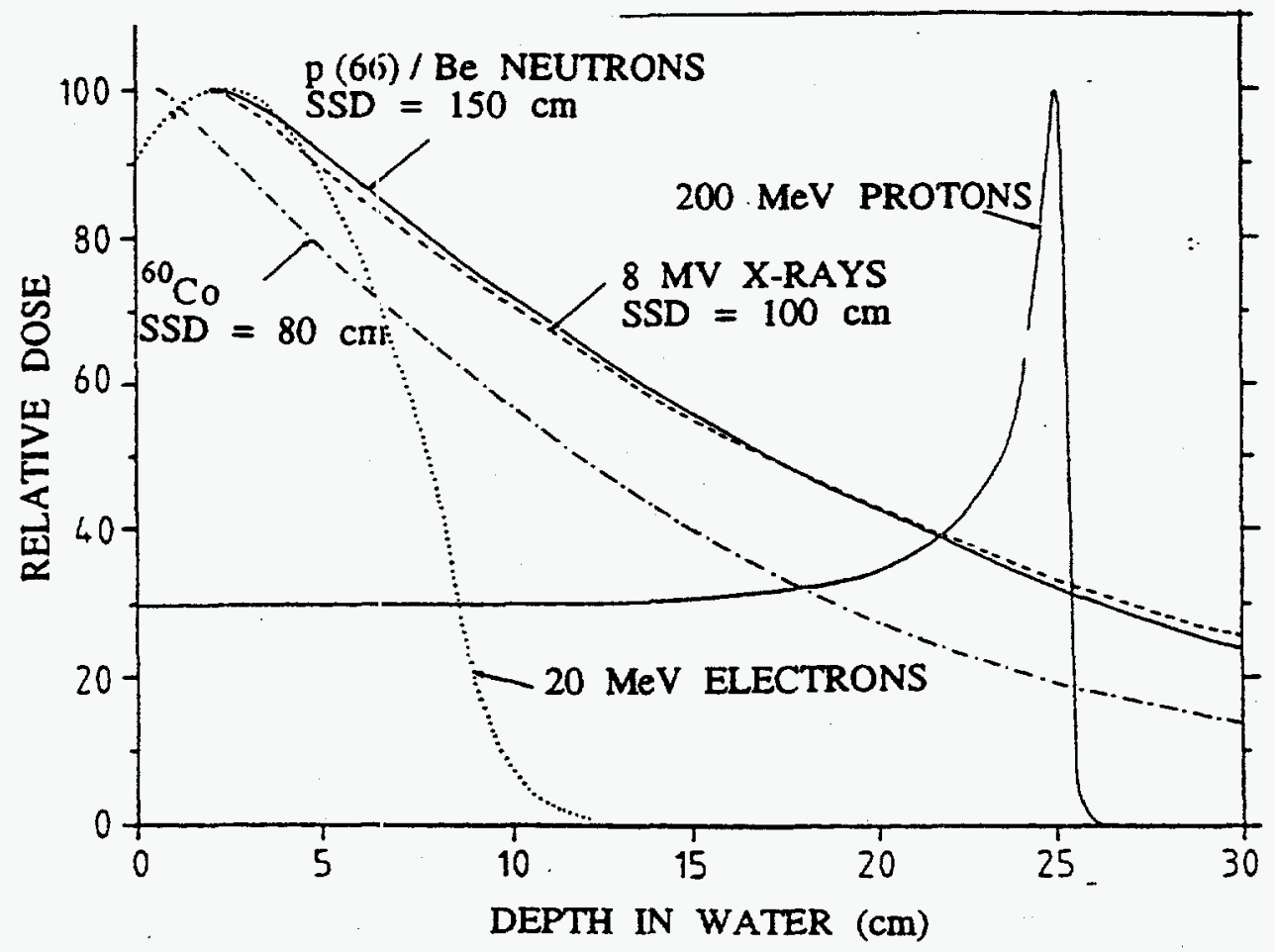

Figure 1. Depth-dose curves for several types of radiation.[2]

In spite of successes of conventional radiotherapy, either alone or in combination with other modalities (surgery, chemotherapy), about 17\% of patients diagnosed with a local cancer (absence of metastases) die due to the failure of the local control; the local recurrence is frequent in sites such as upper digestive tract, brain, skull base, in gynecological tumors and in some tumors with high metastatic rates [9][10]. It is those patients who could in principle benefit from an improvement in radiation therapy. There are two approaches to achieve such an improvement, one through a better dose delivery of conventional radiation and the other through the introduction of new types of radiation for therapy. There are, however, limits to the first approach, one of them being the distribution of the physical dose with depth as mentioned before.

Charged particles heavier than electrons (protons, ions) have properties that make them more attractive for treatment of some tumors [9][11][12]. Their interaction with matter is predominantly through processes involving electrons in target atoms. Because of their much larger mass compared to electrons they suffer significantly less lateral scattering and less beam spreading; this difference becomes more pronounced with an increasing mass of ions. As a heavy charged particle penetrates into the tissue it loses its energy in inelastic processes and its velocity is decreasing. The energy deposition rate is a function of energy and as the particle is slowing down the rate at first increases slowly (Fig.2.) ; this region is usually called a plateau. Toward the end of the path the particle experiences a steep rise in the energy loss rate, just before it is fully stopped (Bragg peak). The position of the Bragg peak deperids on the mass and energy of the particle and on the stopping power of the target. These two effects, a less pronounced lateral scattering and the Bragg peak at the end of the path, led to the first proposal to use protons in radiotherapy about fifty years ago. For tumors with a thickness comparable to the width of the Bragg peak, a charged particle beam with the energy selected such that the peak coincides with the turmor should in principle be capable to deposit a large 
part of its energy into the tumor itself, minimizing the damage to the organs in the entrance channel and avoiding fully any irradiation beyond the tumor. Many tumors, however, have a thickness larger than the width of the Bragg peak, and the target has to be irradiated in several steps, each time with beams of different energy, covering thus the whole volume (Fig.2). This method of the spread-out Bragg peak leads to the tumor conform treatment, applicable in principle to any tumor shape, and representing the closest approach to the ideal procedure.

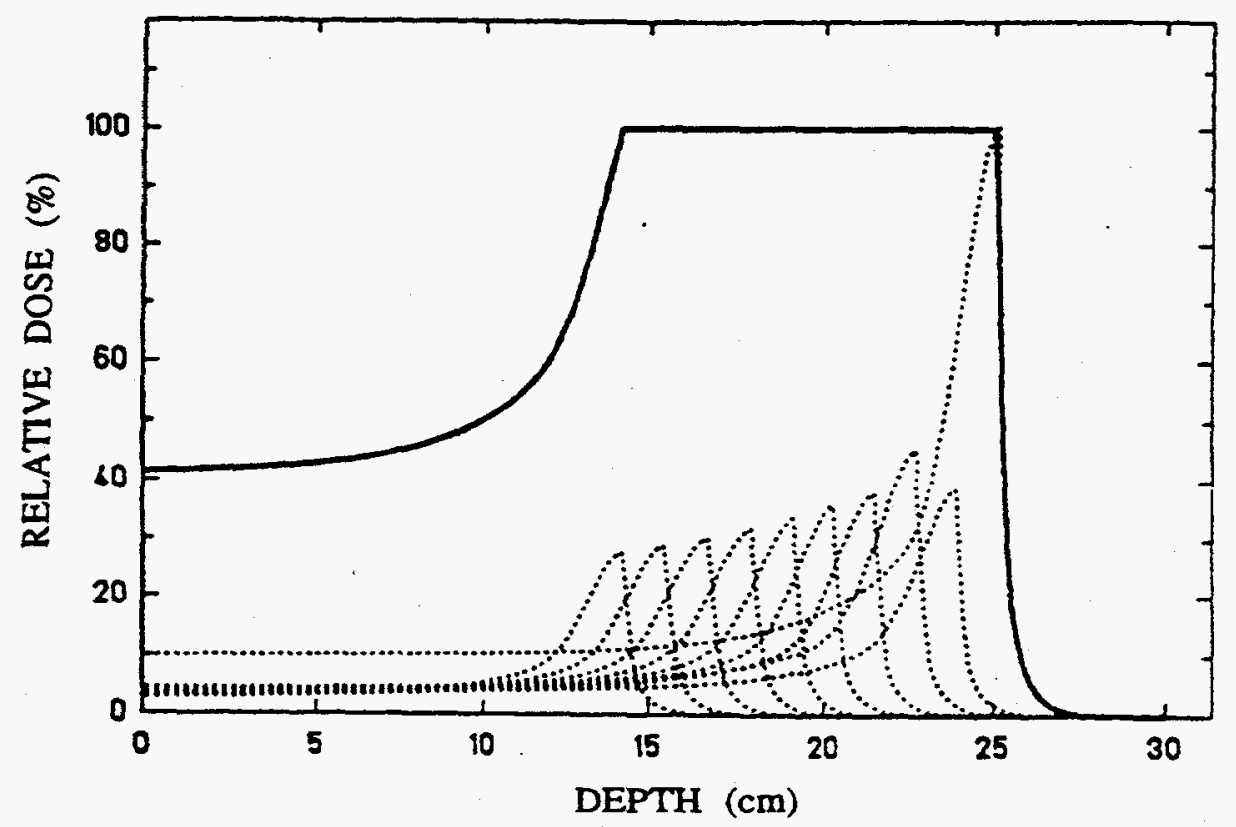

Figure 2. Spread-out-Bragg-Peak (SOBP).[2]

Another parameter relevant for estimating and describing effects of radiation is the linear energy transfer or LET, usually expressed in units $\mathrm{keV} / \mu \mathrm{m}$ [2][11]. Values of this parameter depend on the charge and energy of the particle and therefore change as particles penetrate the tissues. For an ideal monoenergetic beam LET values are meaningful, but for a real beam they are always average values, depending on the way the average has been calculated. Still, this parameter is useful as an indication of the biological effectiveness of radiation and different types are described as being low-LET (photons, protons) or high-LET (neutrons, light ions). The order of magnitude of LET values in $\mathrm{keV} / \mu \mathrm{m}$ is around 1 for photons, between 10 and 100 for protons and up to 1000 for light ions.

The failure of the local control of tumors treated with conventional radiation is in some cases caused by a higher radiation resistance of anoxic cells present in the core of the tumor [2][11][13]. Oxygen enhancement ratio (OER) is a parameter describing this effect; it is defined as the ratio of the absorbed dose of a given radiation to produce a certain biological effect in an anoxic cell population to the dose that would be needed to achieve the same effect in normally oxygenated cell population. OER values for conventional radiation have been found to be as high as 3 , which may indicate the difficulty in delivering a sufficiently high dose to the (anoxic) cells in the core of the tumor, without causing an irreparable damage to the surrounding healthy tissues or critical organs. The oxygen enhancement ratio decreases with increasing LET and for particles with LET values 
above a few hundred $\mathrm{keV} / \mu \mathrm{m}$ it may even approach 1 (Fig.3). Reduced values of the OER have been considered as an important argument for the use of high-LET particles although the clinical studies have not fully confirmed its significance and expectations.

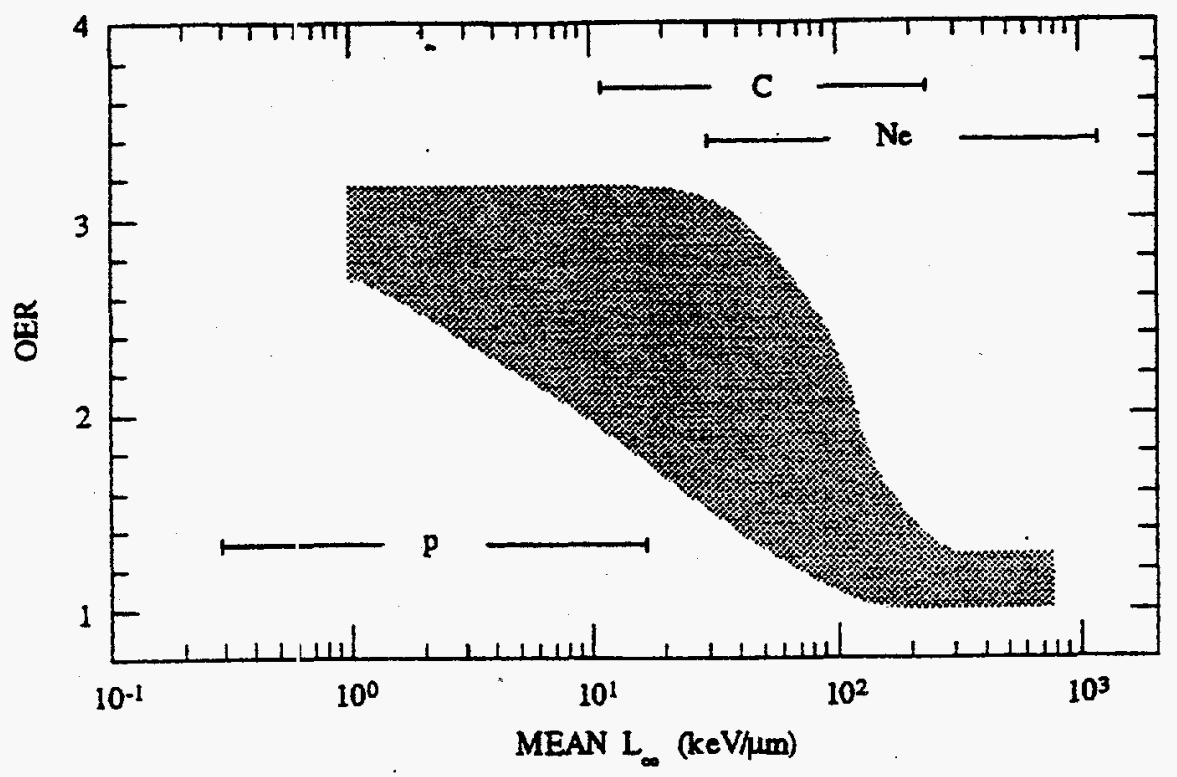

Figure 3. Range of measured OER values, for different types of radiation and cell lines. [2]

Another phenomenon of :mportance for radiobiological effectiveness is the sensitivity of cells to radiation as function of the phase in the cell cycle [11][13]. For conventional radiation cells are most sensitive during the dividing phase while they are more resistant in the DNA synthesis phase. This difference can be very substantial. For light ions, however, the dependence of the sensitivity on the phase in the cell cycle seems to be greatly reduced especially for LET values above a few hundred $\mathrm{keV} / \mu \mathrm{m}$.

While the linear energy transfer-LET describes the energy deposition (or loss) of a particle along its path, the radiation dose is a measure of energy absorbed per unit mass of tissue; the dose is measured in units of Gray or a $\mathrm{ad}(1 \mathrm{~Gy}=100 \mathrm{rad}$ ). This is one of the most important measurable quantities in radiotherapy and it is usually quoted when reporting biological experiments or clinical trials [13][14]. However, equal doses of different types of radiation do not always produce equal biological effects, resulting in different values of the relative biological effectivenes (RBE). The RBE is formally defined as the ratio of the dose of $250 \mathrm{keV} x$-rays to the dose of some other type of radiation, both resulting in the same biological effect. The response of cells, cell populations and tumors in patients to radiation vill vary greatly and a general comparison of RBE values is not very relevant unless all the conditions of the experiment or clinical trial are specified. Still, the general conclusion that the RBE is higher for higher LET (and lower OER) types of radiation remains valid, for the range of interest for ligh.t ion therapy [Fig.4]. 


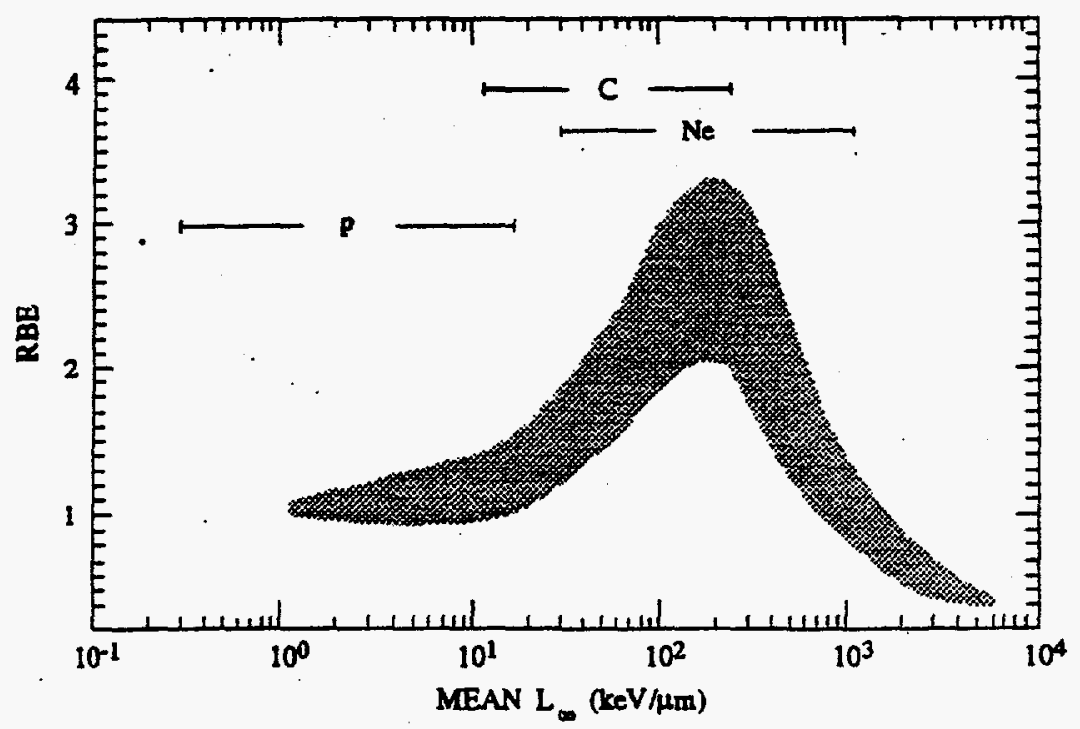

Figure 4. Range of experimental data for the factor RBE as function of LET values. [2]

In summary, protons and light ions have several properties that distinguish them from conventional radiation and offer new possibilities in cancer treatment. For protons the advantages are a better distribution of the delivered dose due to a reduced lateral scattering and due to the existence of the Bragg peak; a reduced entrance dose and no irradiation beyond the distal peak contribute to a more favorable ratio of the doses to the tumor and to the healthy tissues. Biologically, there is no qualitative difference between the conventional radiation and protons but protons have found areas of applications where their characteristics are of fundamental importance for an improved treatment. Light ions experience even less lateral scattering and they have additional characteristics, distinguishing them from both, conventional radiation and protons. While the latter are low-LET types of radiation, light ions are high-LET particles and as such show a reduced oxygen effect, their effects are less dependent on the cell cycle and they have a higher relative biological effectiveness. Light ions therefore could be of benefit in treating slow growing, well defined tumors. However, they have a tendency to fragmentation after a nuclear collision; lighter fragmented particles may have an energy giving them a deeper penetration than the original ion and causing some irradiation of tissues beyond the distal peak. There are also some questions about an increased tumorigenic potential of light ions compared to other types of radiation. It is because of these new effects that the mass range of light ions presently considered for and used in tumor treatment is limited to those below neon; at both facilities where light ions are now used (Chiba, GSI) carbon ions are the species of choice. 


\section{REOUIREMENTS AND PAR IMETERS OF ION BEAMS FOR CANCER TREATMENT}

Ion Species and Energy

Most of the research of the effects of light ions on cells and almost all clinical trials have been done with ions up to neon although the Chiba facility has been designed for ions up to argon. There is a general agreement that carbon ions offer a very good compromise between advantages in the treatment (a very favorable ratio of the dose delivered to the tumor and the entrance dose, good radiobiological properties) and disadvantages that should be minimized (fragmentation, distal dose). For a certain desired penetration depth (or position of the Bragg peak) the energy of ions delivered

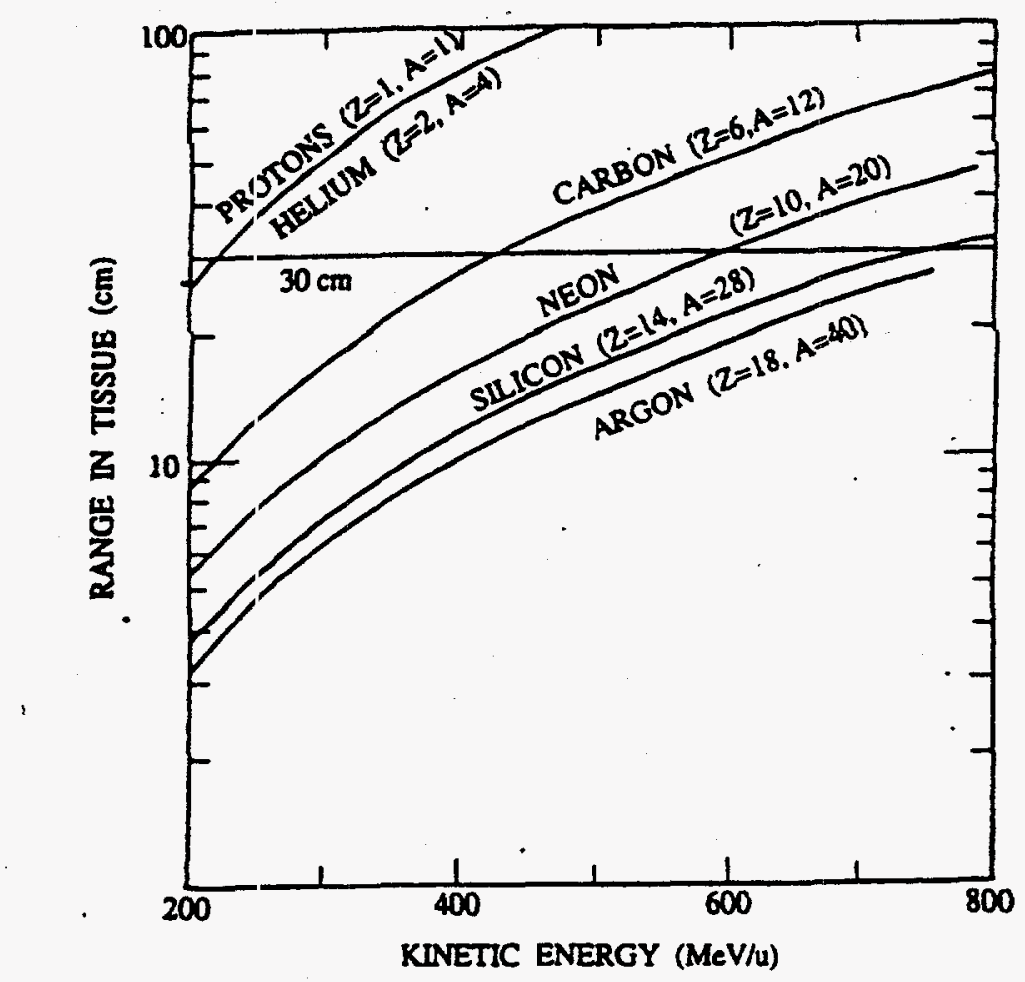

Figure 5. Range-energy curves for several ion species of interest in cancer therapy.[2]

to the patient will depend on the ion species (Fig.5); the ion energy will then determine the size of

the machine and its cost. While for protons an energy of $250 \mathrm{MeV}$ is sufficient for irradiation of tumors seated up to a depth of $30 \mathrm{~cm}$ (water equivalent), light ions require a higher energy for the same penetration. Carbon ions with an energy of $290 \mathrm{MeV} / \mathrm{u}$ will penetrate only $15 \mathrm{~cm}$ deep and for $30 \mathrm{~cm}$ an energy above $400 \mathrm{MeV} / \mathrm{u}$ would be required. For even heavier ions, such as neon, energies about $650 \mathrm{MeV} / \mathrm{u}$ are needed. Once the range of ion species has been selected the top energy of the heaviest ion will determine the size of the machine and therefore its cost as well. There are some trade-offs available in considering those parameters: a machine designed for a certain ion species and the full penetration depth (highest energy envisaged) is capable of delivering even heavier ions 
at the similar or somewhat lower energy per nucleon (the maximum energy will depend on the charge to mass ratio of ions); although for heavier ions the penetration would not be as deep, they could still be used for treatment of those tumors located closer to the surface of the body. For comparison, at an energy of $400 \mathrm{MeV} / \mathrm{u}$ carbon ions would have a penetration depth of $28 \mathrm{~cm}$, oxygen ions $20 \mathrm{~cm}$ and neon ions only $17 \mathrm{~cm}$. Ions like neon and heavier have a relatively higher plateau (dose delivered between the entrance and the tumor) and are preferred for shallow tumor locations to limit the damage to healthy tissues [Fig.6]; a lower energy per nucleon may therefore

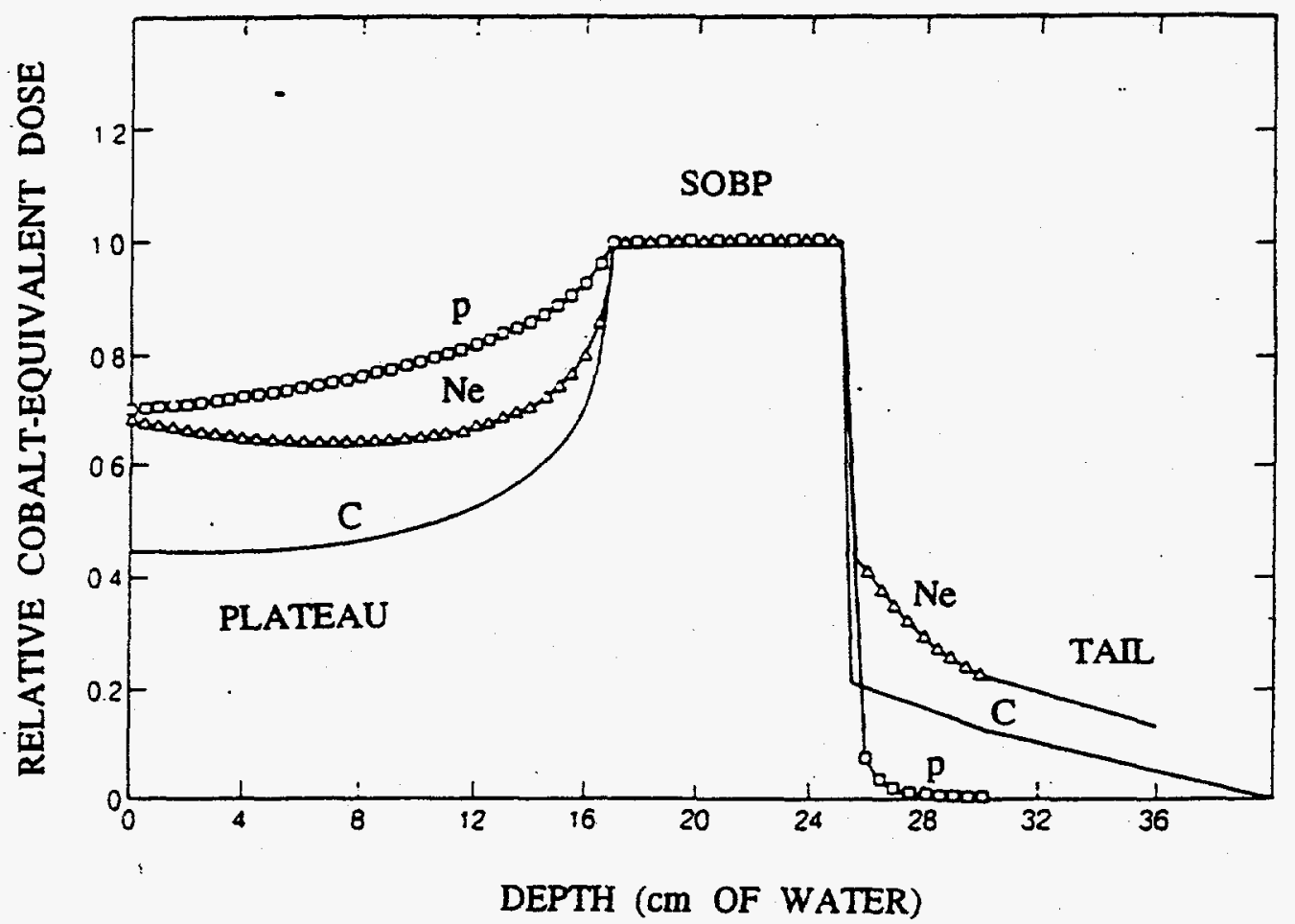

Figure 6. Spread-Out-Bragg-Peak for several ion species, showing relative heights of the plateau and tail regions.[2]

still be satisfactory. Should a deeper range be required, the machine should be designed for a higher energy. There will be additional requirements on the accuracy of achieving the desired energy (position of the Bragg peak) and on the allowed energy spread of the beam (widening of the Bragg peak); it is possible to reduce those two effects by using energy defining collimators in the transport line, but this necessarily results in the loss of beam intensity. However, modern accelerators have already achieved the desired accuracy in energy setting and control $(0.1 \%$ or better); the beam energy spread is also within the limits required for treatment. The knowledge of the properties of tissues in the path of the beam is more critical because this will affect the range (or position of the Bragg peak) and will have to be included into the planning of the treatment.

The treatment of larger volume tumors with protons or light ions requires scanning of the volume with ions of varying energy, to achieve a spread out Bragg peak [15]. There are two methods to achieve the modulation of beam energy, one utilizes a fixed output energy from the accelerator (or, possibly, a few large steps of energy) where the changes of the beam energy when delivered to 
the patient are accomplished by energy degraders in the treatment room (passive systems), while the other is based on the energy modulation of the accelerator itself (active scanning). The first method, exclusively used at present, dces not pose any additional requirement on the accelerator and the beam transfer line except for a fixed and steady output energy, but it does need very carefully designed energy degraders as well as elements for collimation; it may, however, result in a deterioration of the beam quality when passing through the degrader (scattering, fragmentation) and in additional background radiation in the treatment room. For some types of accelerators this is still the only method applicable. The other method, by modulating the output energy of the accelerator itself, moves the burden of complexity from the final part of the transfer line to the machine itself. Modern designs of some accelerators, such as e.g. a synchrotron, and beam transfer lines have reached a stage where it is possible to change the output energy of the machine and necessary transfer line parameters on a pulse-to-pulse basis, which may take only a second or less. This method allows the tumor volume to be scanned a small element by a small element (voxel), always with an appropriate energy and intensity. In spite of the additional complexity of the active scanning, further developments of the accelerator and beam transport control systems will soon make possible the introduction of this method for tests and patient treatment.

Beam Intensity

Beam intensity (or flux), required from the accelerator, is determined by several factors, among them the desired duration of the treatment, prescribed dose, method of beam energy modulation and size and location of the target. To minimize the effects of the motion of the patient during the irradiation it is desirible that treatment times be no longer than at most few minutes (in some cases it may be even necessary to synchronize the radiation pulses with breathing or heart beats). There is some flexibility in the choice of the length of the treatment time; one may e.g. ask for a dose of $5 \mathrm{~Gy} / \mathrm{min}$ to be delivered to a target volume of 2 liters, at the full beam energy. Corresponding values of light ion beam intensities for such a rate of irradiation are of the order of $10^{9}$ particles per second, less lor heavier ion species because of their higher relative biological efficiency. The intensity of the beam at the exit of the accelerator will have to be higher because of losses in beam handling and transport from the machine to the patient. Passive systems in principle will have higher losses, possibly up to $80 \%$, but one cannot expect a transport efficiency much better than $50 \%$ even with active scanuing systems. The latter systems, however, have the advantage that the fraction of the beam, which is not delivered to the patient, is dumped outside the treatment room without contributing to the background radiation. The accelerator, together with the ion source and the injector stage, should be designed for required output beam intensities over the whole range of ion species. For a certain machine design the beam output will be lower for heavier ions but so will be the required target beam intensity. Beam intensities given in Table I should be considered as upper limits; even lower values could be acceptable if this would lead to a simpler and less costly design or to a better beam quality because many tumors are smaller than two liters or a longer irradiation time could be allowed. 
Table I.

\begin{tabular}{|c|c|c|c|c|c|}
\hline Ion & $\mathrm{C}$ & $\mathrm{N}$ & $\mathrm{O}$ & $\mathrm{Ne}$ & $\mathrm{Ar}$ \\
\hline $\begin{array}{c}\text { Beam Intensity on Target } \\
\text { (particles/second) }\end{array}$ & $1 \times 10^{9}$ & $9 \times 10^{8}$ & $7 \times 10^{8}$ & $5 \times 10^{8}$ & $2 \times 10^{8}$ \\
\hline
\end{tabular}

Time Structure of the Beam on the Target

An accurate dose delivery over the volume of the tumor requires a well defined time structure of the beam from the accelerator. If a passive beam delivery system is used, the target is irradiated slice by slice with a broad beam and the time structure is less critical as long as it is possible to monitor and control the time a slice is exposed to the beam. An active beam scanning system poses stricter requirements on the time structure unless there is an on-line beam detection system to accurately measure the dose delivered to any volume element and to deliver a signal to move the beam to the next element once the appropriate dose has been reached. Without such an online system the extracted beam from the accelerator should be as steady as possible, with intensity fluctuations within a few percent only, which leads to very tight tolerances on accelerator and beam line elements and their power supplies.

In addition to the stability of the beam there are other considerations as well. Regardless of the type of the machine, whether it is a cyclotron, linac or synchrotron, the beam will have an inherent intensity structure related to the if accelerating voltage; depending on the type of the machine the beam may be available with a macroscopic duty factor less than $100 \%$. A cyclotron operates with a duty factor of $100 \%$ and there is an if structure corresponding to the frequency of the accelerating voltage; the beam intensity control is done best at the low energy end, ion source or ion injector. A linac usually has a very low duty factor, with pulses of a few milliseconds duration, but a high intensity and, again, showing an if structure corresponding to the accelerating voltage. The low duty factor makes linacs less suitable for ion delivery systems. Finally, a synchrotron with a slow extraction system can have a duty factor up to $50 \%$ and its if structure can in principle be removed by debunching the beam although there are no special advantages in doing this. A synchrotron as a pulsed device is well matched to both, passive and active beam delivery systems. The length of the extracted beam pulse could be made to correspond to the time needed to fully or partially irradiate a slice of the target and the energy can then easily be changed for the next slice. However, the extracted beam intensity in a synchrotron is quite succeptible to any fluctuations or noise in magnet power supplies and efforts have to be made to address this problem.

\section{STATUS OF PROTON AND LIGHT ION CANCER THERAPY, CLINICAL TRIALS}

Facilities and Number of Potential Patients

Statistics of the incidence of cancer and cure rates indicate that there is still room for a substantial improvement. Conventional surgery has reached a high level of safety and further improvements can be expected through the introduction of less traumatic procedures (laser surgery, laparotomies) and a broader use of reconstructive surgery. Chemotherapy is rarely chosen and 
justified as a single modality; patients benefit by its use as an adjuvant treatment. For the local control, most important part of cancer treatment, it is the radiation therapy that contributes greatly to the cure rates and which is still open to new methods of application and to new types of radiation. Even with conventional radiation new techniques such as stereotactic radiosurgery and conformal therapy contribute to an increase of the dose delivered to the tumor without exceeding the allowed dose to healthy tissues. Protons and light ions, however, offer a further improvement in the treatment of certain tumor sites where conventional radiation may often fail. As mentioned earlier, the sites where proton and light ion beams could most benefit the patient are those where their characteristics (physical selectivity, radiobiological effects) can be fully utilized; the preferred sites would be those in a close vicinity of vital organs or those showing resistance to conventional radiation. Experience with proton beams (good physical selectivity), with neutrons (high-LET radiation) and with light ions (good physical selectivity, high-LET radiation) has shown that for a number of tumors substantially better results can be achieved, both in the local control and in the long term survival. On the basis of these characteristics and supported by an experience however limited, estimates have been made for the number of potential cases that could benefit from proton and light ion therapy[2][16][17][18][19]. For the Metropolitan New York area with a population of 20 million it has been estimated that out of 100000 new cancer cases per year about 15000 would benefit from proton therapy; an estimate done for Italy has shown that about 7000 patients per year would benefit from light ion therapy, in a population of about 60 million.In a dedicated facility it should be possible to treat about 1000 to 2000 patients a year; it follows that a proton facility could be fully utilized in any population of about ten million, while a light ion facility would be justified for agglomerations of several tens of million.

The real situation with proton and ion facilities is quite different. Although the use of protons has been suggested fifty years ago, at present there are less than twenty facilities in operation, with less than 20000 patients treated so far. For light ions even less experience exists; fewer than 3000 patients have been treated so far; among them about 2500 in the Berkeley facility which was closed in 1993. Presently there is just one facility, fortunately dedicated, for light ion cancer treatments, in Chiba (Japan), while clinical trials are about to begin in the nuclear laboratory at GSI (Germany). There are three proton facilitie:s projected to be put into operation within a few years; another ion facility in Japan (Hyogo) is projected for the operation in the year 2001 and there are two European projects (TERA; CERN-AUSTRON-TERA-GSI collaboration) in the design stage. This reluctance in embarking with more vigor cn clinical applications of light ions stems not only from the need for a substantial initial investment but also from the uncertainty, expressed by clinitians, that anticipated benefits of light ions in comparison with other types of radiation will not be fully realized in clinical trials.

\section{Clinical Trials with Protons}

Although not the first acility to use protons for tumor treatment, the Harvard Cyclotron Laboratory (HCL) in collaboration with the Massachusetts General Hospital (MGH) has treated the largest number of patients (more than 7000 so far) and gained most experience in the field [12][20] $[21][22][24][25][26]$. Results have been so encouraging that a new machine, again a cyclotron but with a higher energy, will be pu: into operation at the $\mathrm{MGH}$, in 1998. Pathologies treated with proton beams fall into several categories in a certain order of priorities, starting with those close to highly critical structures where advantages of protons have been clearly demonstrated and no additional 
studies are needed; in the second group are pathologies characterized by a prevalently local evolution where local control will lead to a greater probability of cure than by using conventional radiation. Finally protons can be used for palliative treatment of locally advanced tumors with very poor prognosis. At HCL/MGH the largest group of patients have been those treated for ocular tumors, especially uveal melanomas. Treatments have been highly successful with respect to local control, eye retension and visual preservation. A large number of patients with cervical spine and skull base chordomas and chordosarcomas have been treated as well. While the outcome when using conventional radiotherapy and/or surgery for those tumors was very often fatal, a combined photon/proton treatment has resulted in a high local control rate and very good long-term survival rates. Similar encouraging results were achieved with a combined photon/proton treatment of prostate cancer, while the treatment of some intracranial tumors was much less successful.

The other important center in the U.S.A. for cancer treatment with protons is situated in Loma Linda, as a fully dedicated facility [27]. It is a $250 \mathrm{MeV}$ synchrotron, put into operation in 1990 where more than 2000 patients have been treated so far. Anatomic regions treated include brain, head and neck, spine, posterior abdomen and pelvis. The majority of patients were diagnosed with prostate cancer. Together with the MGH facility, Loma Linda synchrotron with its three gantries and one fixed beam will for a long time be in the forefront of proton beam applications in cancer treatment.

At the Paul Scherrer Institute (PSI) in Villigen, Switzerland more than 2000 patients have been treated so far with protons, most of them for ocular tumors with a $72 \mathrm{MeV}$ cyclotron beam [9][28][29][30]. Results were excellent, comparable to the treatment by enucleation but with the advantage of preserving the eye and a reasonable visual acuity, even in unfavorable cases of large tumors. There are plans to broaden the application of proton beams by using the $590 \mathrm{MeV}$ cyclotron at PSI; the energy will have to be reduced to match the required range even for treatment of deep seated tumors. It is envisaged that the tumor volume will be scanned in three dimensions, moving the Bragg peak longitudinally by using a number of range shifters, sweeping magnetically the beam in one perpendicular direction and slowly moving the patient in the other.

The Proton Medical Research Center at Tsukuba, Japan [31] has concentrated its efforts on treatment of thoraco-abdominal tumors, a different emphasis from other similar centers. About 500 patients have been treated thus far, using the $500 \mathrm{MeV}$ synchrotron beam, degraded to $250 \mathrm{MeV}$. Due to a relatively small number of cases per type of tumor the results are not conclusive but there are indications that primary malignancies of the esophagus, lung and liver may benefit from the improved physical dose distribution of proton beams, when applied either alone or in combination with photons.

There has been an active program of proton therapy in Russia since 1969, mostly at the Institute for Theoretical and Experimental Physics (ITEP) in Moscow [32][33]. Close to 3000 patients have been treated so far for a large variety of tumor sites, many of them in an inoperable stage, by using the $70-200 \mathrm{MeV}$ proton beam from the ITEP synchrotron. Although the number of cases per site has been rather small and therefore the statistics are not reliable, results for some tumors have been very encouraging, in a good agreement with clinical trials at other centers. The main difficulty for the clinical work in Russia has been the lack of dedicated facilities; medical applications usually have had a much lower priority in scheduling the time on the machine. The prospects for the recently prepared program to develop a hospital based facility are, however, not very good because of the present economic situation in Russia. 


\section{Clinical Trials with Light Ions}

In spite of expected benefits of light ion radiation, due to their better physical selectivity and to additional radiobiological effects, there have been very few clinical trials worldwide, with a very limited number of patients. If trials with helium nuclei are excluded from consideration (helium nuclei have characteristics similar to protons, i.e. they are low LET radiation), there were only about 500 patients treated so far, with either neon or carbon ions. The pioneering work was done at the Lawrence Berkeley Laboratory, in the period between 1979 and 1992 when about 300 patients were irradiated with neon ions from the Bevatron facility; the facility was closed soon thereafter and no further trials were done [34][35][36][37][38][39]. The most commonly used energy of neon ions was $585 \mathrm{MeV} / \mathrm{u}$, sufficient to penetrate to the deepest tumors. Most patients were selected for neon ion treatment because conventional modalities were ineffective (inoperable tumors, tumors not responding to conventional radiation). This fact makes a comparison more difficult because easily treatable cases, with a more favorable outcome, were excluded. The objective of those trials was to develop techniques for therapy planning and delivery, study the response for a variety of tumors and evaluate the acute and late toxicity of neon irradiation. The list of treated tumor types is long and, therefore, the number of patients was too small for good statistics. Still, neon ions appear to offer potentially improved local control and survival rates for a number of tumor types; results agree well with those obtained with other high-LET radiation such as neutrons. Improved control and survival rates were achieved for patients with paranasal sinus tumors, some salivary gland tumors, bile duct carcinomas, some soft tissue and bone sarcomas, and advanced prostate carcinomas; in some cases the rates were twice as high as with photons. The outcome of treatment of other types of tumors, such as some brain tumors, melanomas, advanced head and neck tumors, lung, esophageal, gastric and pancreatic malignancies, was not better than with conventional therapy, which usually meant that it was not favorable. The conclusion of the LBL trials was that neon ion radiotherapy offers a clinically feasible modality for several selected human cancers, with improved results when compared to conventional photon therapy. It is expected that better results, with fewer side effects, could be obtained with a better, more conformal system of beam delivery than used at LBL.

Although all patients treated at the LBL with light ions (excluding helium) received neon ion therapy, this choice does not seem to be the best. Carbon ions have radiobiological dose distribution features which have advantages compared to both protons and neon ions. While the physical selectivity is similar for all those particles, carbon ions have high-LET characteristics, and compared to neon ions, a lower dose in the plateau region and a smaller fragmentation tail beyond the target. The two facilities where light ions are used (or soon will be) have decided to concentrate the trials on carbon ions although the range of available species is much broader. The first facility is at Chiba, where clinical trials have been in progress since 1994 and about 200 patients have been treated with carbon ions [40][41][42][43]. A wide variety of tumors have been and will be treated, including some deep-seated tumors which did not respond well to neon beams. Patients are carefully selected according to a number of criteria to determine the tumor response and the toxicity for normal tissues; locally advanced and/or inoperable localized carcinomas have been chosen. For head and neck sites locally advanced, recurrent or radioresistant tumors not treatable with other modalities are selected; brain tumors selected for treatment are malignant glioma and astrocytoma, while other sites include inoperable lung tumors, primary liver tumors, cervical cancer, prostate cancer, esophageal cancer and inoperable sarcomas of the bone and soft tissue. In the first phase it is intended to use conservative doses, below those tolerated by healthy tissues; later on the dose will be increased in small 
increments. This approach is justified because of the need to establish proper protocols for the treatment and because of high values of the factor RBE for some tissues such as the central nervous system which have to be spared as much as possible. Preliminary results show that there were no major healthy tissue morbidities and that carbon ion therapy is a promising modality for cancer treatment. Trials are planned to continue, expanding eventually the range of ion species to silicon or argon (for tumors located closer to the surface), with the hope to determine the appropriate role of ions in radiotherapy.

At the GSI heavy ion research complex in Darmstadt (Germany) an experimental cancer treatment program is underway, to continue for five years and to include about 350 patients [44][45]. The main objective of the program is to test a novel, most advanced method of beam delivery by using two-dimensional magnetic raster scanning coupled with an active accelerator energy modulation. An ionization chamber in front of the patient measures the number of ions at a specific point in the tumor volume and controls the speed of scanning. After the successful conclusion of the clinical trials the plan is to design and build a hospital based facility.

\section{TYPES OF ACCELERATORS FOR LIGHT ION THERAPY}

\section{Cyclotrons}

Cyclotrons are machines with a constant magnetic field and a fixed frequency of the accelerating voltage. The injection of the beam from the ion source, its acceleration in the machine and ejection are a continuous process; the extracted beam has a fixed energy and its intensity can also be continuous which can have advantages when scanning the tumor. While cyclotrons delivering proton beams with energies up to $230 \mathrm{MeV}$ have already been developed by industry to operate in a hospital environment, their application as accelerators for light ion therapy is not very feasible. The energy per nucleon needed for the same penetration depth is higher, also the charge to mass ratio of ions compared to protons is lower; because of these factors a standard design light ion cyclotron for cancer treatment would have a prohibitively large magnet. The only cyclotron even considered for light ions was part of the now abandoned EULIMA (EUropean Light Ion Medical Accelerator) project. In order to reduce the large size and weight of a standard magnet, a superconducting single coil design was considered and developed for EULIMA, having an external radius of only $2.32 \mathrm{~m}$. However, the cyclotron was not the preferred choice for this facility because the required superconducting technology was very sophisticated, not justifying other advantages of a cyclotron.

\section{Linear Accelerators}

Conventional linear accelerators are usually very low duty factor machines, delivering high ion beam currents in short pulses (millisecond or so duration), often for injection into the next stage accelerator such as a synchrotron. They can accept and accelerate ions having a certain ratio of the charge to mass and deliver a beam with an energy fixed or, at best, variable in large steps. Although the extraction efficiency is close to $100 \%$, there are presently no linear accelerators used for either proton or light ion therapy (there was a proposal to use a small fraction of the proton beam from the Brookhaven National Laboratory's $200 \mathrm{MeV}$ linear accelerator for cancer treatment but it was decided not to proceed). Linear accelerators are machines requiring a large space, they are expensive 
to build and to maintain and beam characteristics are not most favorable for radiotherapy. Improved performance (broader spectrum of ion species, a higher duty factor, some flexibility in output energy, reduced size) can be achieved by using superconducting cavities but this is again a sophisticated technology which is not very suitable for a hospital environment.

Synchrotrons

A synchrotron is a pulsed accelerator, with particles moving on a closed, approximately circular trajectory where the magnetic field and the frequency of the accelerating voltage vary in time as the energy of particles increases. The pulse rate of a synchrotron is of the order of 1 second or less except for very large machines, and the duty factor can be as high as 50\%. The energy of the extracted beam depends on the linal value of the magnetic field and can be changed on a pulse-topulse basis, which makes this type of a machine very well matched to the depth scanning by beam energy modulation. Although the extracted beam intensity is lower than from either a cyclotron or a linear accelerator, by a proper design it can be made sufficiently high for any ion species and for treatment of tumors at any depth. Other extracted beam parameters, such as emittance, energy spread or time structure, can also be matched to the needs of the beam delivery system. Due to its advantages, flexibility in output energy and ion species, a sufficient intensity, a reliable operation and a moderate size and cost, al synchrotron is the machine of choice in all projects for light ion treatment of cancer.

\section{LIGHT ION FACILITIES. EXISTING AND FUTURE}

History: BEVALAC Program

Although the first acceleration of ions heavier than helium was accomplished in 1971, clinical trials had to wait until 1975, for the completion of the BEVALAC complex consisting of the synchrotron Bevatron and the linear accelerator SuperHILAC serving as its injector. The beam resources of this facility were shared between the nuclear physics research and biomedical studies. There were two treatment rooms, both utilizing horizontal beams. Initially a scattering system with two foils was used to spread the beam but uniform fields larger than $20 \mathrm{~cm}$ diameter were difficult to achieve without a significant deterioration of beam properties. In 1983 a magnetic wobbler system was installed consisting of two orthogonal dipole magnets and delivering the beam in a set of concentric circles, their radii controlled by the amplitude of the magnet coil currents. A more advanced raster scanning system was subsequently developed and commissioned just before the shutting down of the facility in 1993. In the retrospect the main drawbacks of the BEVALAC program were the difficulties in running a machine for two different programs, with different requirements, rather high operating. costs, beam characteristics not optimized for medical applications and a relatively high incidence of breakdowns which is not acceptable for routine radiotherapy. 
Loma Linda Proton Synchrotron

Although this machine was not designed for light ions, the review of existing facilities will begin with the proton synchrotron at the University at Loma Linda because it is the first synchrotron built for a hospital and used exclusively for medical applications [46][47]. The engineering design and fabrication of the accelerator and beam transport systems were done by the Fermi National Accelerator Laboratory (Fermilab), beginning mid-1986. The maximum energy has been chosen to be $250 \mathrm{MeV}$, sufficient to treat even the deepest tumors. A duoplasmatron proton source feeds a 2 $\mathrm{MeV}$ radio frequency quadrupole (RFQ) which serves as an injector into the synchrotron. The machine is weak focusing, which made it simpler but it may have limited the intensity. In the year 1990 the first patient was treated, and since then the facility has been fully commissioned to include three gantry rooms and one fixed (horizontal) beam room. The operation of the machine itself has been very satisfactory, it was stable, reliable and reproducible. It will be very interesting to compare the experience with this facility with the MGH new cyclotron facility, soon to become operational, to see whether a single approach in the design of a proton facility is sufficient or whether both should be pursued. As a reference, it can be mentioned that Loma Linda synchrotron could accelerate heavier particles, such as fully stripped ions from helium up to possibly neon, but with a much lower intensity and a final energy just below $70 \mathrm{MeV} / \mathrm{u}$, which would be barely sufficient to irradiate even tumors very close to the surface.

HMMAC Facility (Chiba)

The Heavy Ion Medical Accelerator in Chiba (HMMAC) is the first and still the only light ion accelerator in the world which has been designed specifically for medical and radiobiological applications [48][49][50][51][52]. The parameters of the machine were defined broadly enough to cover possible future requirements for heavier ion species and a higher maximum energy. The range of available ions goes thus from helium to argon, and the maximum energy was chosen to be 800 $\mathrm{MeV} / \mathrm{u}$ for silicon, sufficient for a penetration depth of $30 \mathrm{~cm}$ ( for argon ions the maximum energy is $700 \mathrm{MeV} / \mathrm{u}$, which is sufficient for their optimal application, treatment of tumors close to the surface). The required beam intensity was determined on the basis of the dose rate of $5 \mathrm{~Gy} / \mathrm{min}$, for a field size of $22 \mathrm{~cm}$ diameter. This dose rate requires intensities ranging from $2.7 \times 10^{8}$ particles per second (pps) for argon to $1.2 \times 10^{10} \mathrm{pps}$ for helium; for carbon which is presently used for clinical trials the required intensity is $2 \times 10^{9} \mathrm{pps}$. A synchrotron is the only accelerator capable to satisfy the requirements for such a broad range of ion species, beam intensities and energy. In order to achieve such a flexibility and increase the reliability of the facility it was decided to build two synchrotron rings, one on top of the other. The upper ring was designed for a somewhat lower energy, $600 \mathrm{MeV} / \mathrm{u}$ maximum, and it delivers a vertical beam to two treatment rooms and a horizontal beam to a room for radiobiological studies. The lower ring delivers a horizontal beam at the full energy of $800 \mathrm{MeV} / \mathrm{u}$ to two treatment rooms as well as a beam for general studies. Because of a much higher rigidity of light ions to be used in therapy compared to protons (for the same magnetic field the radius of curvature has to be larger by a factor of about 4 for heaviest ions and highest energy) gantries for light ions would have to be larger by a similar factor and most often are not considered for use in light ion facilities.

The detailed design and arrangement of the elements of the injector were determined by the beam parameters of available ion sources. There are two ion sources used in the injector, a Penning 
source with a hot cathode suitable for ions from helium to neon and an Electron Cyclotron Resonance (ECR) ion source for elements up to argon. Installation of an upgraded ECR ion source is underway, to provide the facility with even heavier ions, such as Fe. The two selected sources do not produce fully stripped ions, which is a requirement for acceleration in the HIMAC synchrotron; ions have to be first accelerated to an energy high enough to achieve an efficient stripping to bare nuclei. The first stage of acceleration is done in an RFQ accelerator, designed to accept ions in a low charge state (relative charge to mass ratio $\mathrm{q} / \mathrm{m}>1 / 7$ ); such a low value of the ratio $\mathrm{q} / \mathrm{m}$ dictates that the RFQ has to be very long $(1=7.3 \mathrm{~m})$ which complicates its design. The output energy of the RFQ is still not high enough to achieve a full stripping and ions are fed into a $24 \mathrm{~m}$ long Alvarez type linear accelerator where their energy is raised to $6 \mathrm{MeV} / \mathrm{u}$; this is sufficient to achieve a high fraction of bare nuclei when the beam passes through a thin carbon stripping foil. The size of the ring is primarily determined by the final, maximum ion energy and at HIMAC the circumference is $130 \mathrm{~m}$. The focusing is strong, of the sieparated function type. The maximum dipole magnetic field is $1.5 \mathrm{~T}$, with a rise time up to $2 \mathrm{~T} / \mathrm{sec}$. Repetition rate can be varied between 0.3 and $1.5 \mathrm{~Hz}$, with a flat top of up to $400 \mathrm{~ms}$. For acceleration of heavier ions (Fe) the two rings could be operated in cascade.

The beam delivery system is standard, with two orthogonal wobbler dipole magnets to cover the required target field, a scatterer to achieve a smoother transverse dose disribution, a ridge filter to broaden the momentum spread and thus the Bragg peak, and a range shifter to change the beam energy. Although the synchrotron energy can in principle be varied on a pulse-to-pulse basis, the necessary instrumentation and controls have not yet been installed and the energy is varied by means of range shifters. The maximum field size is $15 \times 22 \mathrm{~cm}^{2}$.

The Chiba facility is part of the national program to combat cancer, developed in 1984 . The construction started in 1988, machines were commissioned in 1993 and clinical trials started the following year. The cost of the facility was high, more than 300 million dollars, with yearly operating costs of 45 million dollars. The whole system operates well, reliably and reproducibly; at present the most important improvement project deals with removing ripples and fluctuations in the extracted beam.

\section{GSI Facility}

Use of light ions from the GSI synchrotron SIS in radiotherapy was considered at the time of the proposal for the machine itself, but its realization had to wait until early 1990's [44][53][54][55]. At that time there was already some experience gained at LBL with its BEVALAC facility, showing better results in treatment of several tumors compared to conventional radiation. Although light ions, which are high-LET radiation, should have certain advantages with respect to low-LET protons, clinical results from LBL did not fully confirm that; as one possible explanation it was suggested that the dose delivery system at LBL has permitted an essential part of this highLET radiation to be depositel outside the tumor, thus limiting the dose to the tumor itself. The objective of the GSI program has been to develop the best possible conform dose delivery system and to test the effects of light ions under such conditions. The machine itself has not been designed with medical applications in mind, but for production of all ions up to uranium for nuclear physics studies. The ring is rather large, and the maximum energy reaches $2 \mathrm{GeV} / \mathrm{u}$ for particles with $\mathrm{q} / \mathrm{m}$ $=0.5$ or $1 \mathrm{GeV} / \mathrm{u}$ for uranium. The ion beam spill may be as long as 1 to 2 seconds. Recent upgrades had as the objective an increase of intensity to the space charge limit of the ring but the original light ion intensity was already sufficient for tumor treatment. The linear accelerator UNILAC serves as 
the injector into the ring; this was a logical solution because UNILAC was already available and in operation.

Of the two basic approaches in the design of the beam delivery system, the active beam spreading method was selected. The other approach, a passive system, has the disadvantage of irradiating a substantial fraction of healthy tissues around the tumor with potentially lethal high-LET ions. In the passive system the ratio of the doses to the tumor and surrounding tissues can be improved by a careful design of a number of beam shaping modules for each patient, depending on the shape of the tumor and energy of the beam for a particular slice of the target; this process is demanding and costly. A great deal of effort was devoted to studies of active systems at GSI. In principle this is a simple system: the treament volume is dissected into slices of equal thickness and each slice is irradiated separately by moving the beam across its cross section. The shapes of consecutive slices will be quite different one from the other, but using the active beam delivery it should be possible to treat tumors of any shape. The control of beam energy to match the depth of a slice is done by modulating the accelerator and beam transfer line parameters.

To scan the beam laterally, across a slice, two different methods have been considered, raster and voxel scanning. In the raster scanning the beam is moved continuously over the slice, and the writing speed is adjusted according to the required dose for that particular spot. In the voxel scanning the beam stays at a spot long enough to deliver the required dose, then it is turned off and moved to the next spot. In the practical realization there are no fundamental differences between the two methods and they both are capable of creating the proper dose distribution. While the motion of the beam across the slice is in principle a matter of controlling the elements of the beam transfer line to match the shape of the slice, it is much more complex to properly deliver the needed dose for each volume element. First, the beam with its energy adjusted to place the Bragg peak into the distal slice will deliver a dose, although lower, to all slices closer to the surface. The dose to be delivered to any subsequent slice has to take into account what has been previously deposited. Second, light ions are subject to fragmentation when passing through the matter and those lighter fragments may have a deeper penetration, delivering a certain dose beyond the Bragg peak, i.e. in the healthy tissue beyond the tumor (Fig. 6). Third, the relative radiobiological efficiency will be a very complex function of many parameters, such as particle energy, nuclear fragmentation, and properties of different tissues; it will have to be estimated in the best possible way to determine what dose to deliver to each volume element and then properly instruct the beam delivery system. The last problem remaining is to measure on-line the dose that has been delivered to a certain spot and then give the signal to move the beam; fast on-line ionization chambers are used for that purpose. On-line control of the dose distribution has an additional advantage: the system will be much less sensitive to ripple or fluctuations in the extracted beam intensity which was of primary concern for passive systems.

The extracted beam energy is changed by varying the parameters of the machine; a large number of fixed energy values have been selected and corresponding machine settings stored in a computer, to enable a pulse-to-pulse change of energies. The approach selected by the GSI is evidently the most advanced and should be capable of adjusting the delivered dose to the shape of the tumor with the minimum damage to the healthy tissues. In a few years when the first results are known, it will be possible to compare not only the passive method as used at the HIMAC with the GSI active method, but also to determine whether a better dose delivery will prove expected advantages of light ions.

A few years ago, as an exercise, a medical synchrotron for light ions was designed at GSI. The maximum energy was $480 \mathrm{MeV} / \mathrm{u}$, for all species up to neon; the intensity was chosen rather low, 
$10^{8}$ neon ions per second, resulting in a smaller vacuum chamber. The size of the machine was also relatively small, about $50 \mathrm{~m}$ in circumference.

\section{COSY Facility}

The cooler synchrotron and storage ring COSY, recently put into operation at Julich, Germany, has also been considered for medical applications [56][57]. Although primarily a proton machine, intended for nuclear physics, it should be capable to accelerate light ions up to neon. At the maximum magnetic field the energy of light ions would be similar to that of the HIMAC facility but substantial modifications of the injector would be required to expand the range from protons to light ions. There are several areas of studies covered by the proposed medical program, among them comparison of active and passive beam spreading systems, treatments with a fixed horizontal line vs. a rotating gantry, and as a long range objective, the comparison of protons and light ions. As this facility will remain primarily a nuclear physics center, the number of patients will be limited to about 100 per year if at some future time clinical trials begin.

\section{TERA Project}

The TERA project is an ambitious study by a large collaboration of Italian institutions, universities and hospitals with the goal of establishing an Italian center for hadrontherapy (therapy with protons, neutrons and light ions) [2][3][58]. The initial goal has been later broadened to form a whole network of facilities for hadron cancer treatment, called RITA. This network would consist of the oncological hadrontherapy center, linked with several centers devoted to proton therapy and with other hospitals. For the oncological center, the study concluded that the best option would be a synchrotron serving for acceleration of $\mathrm{H}^{-}$and light ions. Protons within an energy range of 60 to $250 \mathrm{MeV}$ would be generated at extraction, by stripping of accelerated $\mathrm{H}^{-}$ions. The same ring could be upgraded in the future to accelerate fully stripped light ions up to oxygen, at energies between 120 and $400 \mathrm{MeV} / \mathrm{u}$. When operating with $\mathrm{H}^{-}$ions, the ion source will be followed by an RFQ with the output energy of $2 \mathrm{MeV}$; the beam will then be further accelerated in a linear accelerator up to an energy of $11 \mathrm{MeV}$ and injected into the synchrotron. For light ions the choice of the ion source is of crucial importance for the design of the injector itself. Two ion sources have been considered, a Penning ion source delivering high ion currents but in low charge states, and an ECR ion source with a lower yield but higher charge states. The first injector design was based on a Penning source delivering $\mathrm{O}^{2+}$ ions; this would be followed by an $\mathrm{RFQ}$ to raise the energy to $250 \mathrm{keV} / \mathrm{u}$. The final stage, a synchrotron, needs fully stripped ions for injection and low charge state oxygen ions have to be pre-accelerated to an energy high enough to achieve the full stripping. For the TERA project the process of raising the charge state would proceed in two steps. After the initial acceleration in the linear accelerator to an energy of $850 \mathrm{keV} / \mathrm{u}$ the optimum charge state after stripping is $\mathrm{O}^{6+}$; this will be followed by further acceleration to $3 \mathrm{MeV} / \mathrm{u}$, sufficient for a good yield of fully stripped oxygen ions. This is a rather complex scheme and not very efficient because it requires a two-section linear accelerator, with one stripping foil inserted between the sections and one after the second section. Altough the yield of the optimum charge state after the stripping foil is much lower than the beam intensity before the foil, it is expected that the synchrotron output would be sufficient to deliver the required dose to the patient. Both injectors, $\mathrm{H}^{-}$for proton therapy and the light ion injector, would require about the same injection magnetic field which simplifies the operation. The 
design value of the maximum magnetic field needed for acceleration of fully stripped oxygen ions to an energy of $400 \mathrm{MeV} / \mathrm{u}$ is $1.4 \mathrm{~T}$; for the maximum energy of $\mathrm{H}^{-}$ions of $250 \mathrm{MeV}$ the field is only $0.537 \mathrm{~T}$ which allows an efficient acceleration with very small losses due to stripping of $\mathrm{H}^{-}$ions in the magnetic field. The proposed lattice is of the strong focusing, separated function type, with a circumference of about $60 \mathrm{~m}$. The repetition rate is $2 \mathrm{~Hz}$ for $\mathrm{H}^{-}$operation and $1 \mathrm{~Hz}$ for light ion operation; the flat top is $0.3 \mathrm{~s}$.

The oncological center has been designed to have five treatment rooms, two with proton gantries capable of handling $250 \mathrm{MeV}$ beams, one room with full energy horizontal and vertical proton beams, one room with two lower energy horizontal beams for treatment of eye and head and neck tumors, and one room devoted to future light ion therapy; there will also be an experimental room for protons and light ions. When fully in operation, about 1000 patients could be treated per year. At this stage of the project both, passive and active beam delivery systems are under consideration. It should be mentioned that as part of the TERA project other options for proton acceleration have also been considered, such as a compact synchrotron and a compact high frequency linear accelerator.

Hyogo Project

In addition to the HIMAC facility, which has been put into operation a few years ago, there is another proton/light ion facility under construction in Japan. This is the Heavy Ion Medical Accelerator Project by Hyogo Prefecture Government [59], planned for initial operation in the year 2001. The facility will use protons, helium and carbon ions, with energies up to $230 \mathrm{MeV} / \mathrm{u}$ for protons and helium and up to $320 \mathrm{MeV} / \mathrm{u}$ for carbon. Beam intensities have been determined from the requirement for a dose rate of $5 \mathrm{~Gy} / \mathrm{min}$ delivered to a target volume of $15 \mathrm{~cm}$ diameter and a fully extended spread-out Bragg peak. Ion beam energies will allow a penetration depth of $30 \mathrm{~cm}$ for protons and helium ions and $20 \mathrm{~cm}$ for carbon ions. The repetition rate of the synchrotron is $1 \mathrm{~Hz}$ for protons and $0.5 \mathrm{~Hz}$ for other ions, with a spill length of $0.4 \mathrm{~s}$. There will be five beam lines, three for helium and carbon ions (one horizontal, one vertical and one $45 \mathrm{deg}$. oblique line) and two proton lines with gantries. For the initial operation a passive beam delivery system is envisaged.

\section{Med-AUSTRON Initiative}

The initiative Med-AUSTRON was established in 1995 with the objective to study the feasibility for a proton and ion cancer research center in Austria. Studies are ongoing, in collaboration with the TERA Foundation, CERN and GSI, and the results will be presented at a meeting scheduled for October 1997. Preliminary parameters of the ring are slightly different than in the original TERA proposal, although the machine is still designed for protons and light ions. The light ion species considered in this study is carbon (as presently used at Chiba and GSI facilities), with a maximum energy of $425 \mathrm{MeV} / \mathrm{u}$. The ring has a larger circumference, $71 \mathrm{~m}$, but this increase in size would add very little to the overall cost of the facility. In addition to the studies of slow beam extraction from the synchrotron, there are a number of related issues to be covered as well, such as beam stability during the spill and passive and active beam delivery options. 


\section{BNL Booster}

At Brookhaven National Laboratory, Upton, U.S.A., there is a $200 \mathrm{MeV} \mathrm{H}^{-}$linear accelerator; it was recently proposed to use a small part of the beam for proton therapy but the plan was abandoned because of difficulties in scheduling the two applications, high energy physics and medical, without one interfering with the other [17]. Another accelerator, a Booster synchrotron, was designed and constructed to serve as injector of any ion species (protons to uranium ions) into the Alternating Gradient Synchrotron (AGS). The maximum energy as well as beam intensities have been determined by the needs of the AGS ring (and further, of the Relativistic Heavy Ion Collider RHIC, presently under construction) and are more than sufficient for any medical application.For light ions such as carbon or oxygen the AGS Booster is capable, using the existing Tandem van de Graaff injector, to provide a sufficient beam intensity at any energy required for tumor treatment. Other ion species (nitrogen, necn) would need a new ion source and injector to replace the tandem. There is a proposal to utilize the Booster beam for radiobiological studies of interest to NASA with ions up to iron, but patient treatment has not been included in the proposal. By adding a new ion source and injector it would te possible to extend the range of parameters of the Booster (ion species, energy, intensity) and change them on a pulse-to-pulse basis. The interference with the principal mode of operation of the Booster, which is acceleration of ions for injection into the AGS, would be minimal because AGS and RHIC will need the beam only part of the time.

\section{A DEDICATED LIGHT ION FACILITY FOR CANCER TREATMENT}

\section{Accelerator}

The analysis of light ior beam parameters, required for tumor treatment, has shown that of the three types of accelerators considered in this report it is only a synchrotron that is capable to deliver beams of different species, with an energy variable on a pulse-to-pulse basis and a duty factor of the extracted beam well matched to either the passive or active mode of beam delivery [60][61]. A cyclotron is a machine designed for a fixed energy and range shifters have to be used if lower energies are needed; this may result in a deterioration of beam properties, one of the most important characteristics of light ions. Conventional cyclotron designs when extended to light ions lead to very large and massive machines and superconducting magnets have to be considered instead. Even this technology requires large units and a very sophisticated design which is not very suitable for a hospital environment. A linear accelerator for ion energies of interest in tumor therapy is a very long, expensive machine; although it is in principle possible to design a linear accelerator for handling different ion species and even to modulate its final energy, there are other disadvantages that preclude this option. On the other hand, a number of synchrotrons have been constructed so far, at an acceptable cost, for differint purposes and a broad range of parameters (ion species, energy, duty factor, duration of the flat top). They perform well, reliably and with a good reproducibility and they are accelerators of choice for light ion tumor therapy.

The synchrotron lattice designs have been perfected to a high degree and at present it is possible to find a design close to the optimum for given beam parameters. The expertise for a proper design exists at many laboratories around the world and industry may be willing to participate in the 
construction, in collaboration with one or more laboratories. Existing designs, such as TERA or Med-AUSTRON, can serve as the basis for any new light ion facility.

\section{Ion Sources}

There is, however, one element of the accelerator with some room for improvement. This is the preceding stage, the injector. Several considerations require that only fully stripped ions be injected into the synchrotron. First, the acceleration efficiency depends on the charge state of ions, therefore the size of the machine, its cost and time for ions to reach the final energy will also depend on the charge state; this is why the injector into the synchrotron has to produce fully stripped ions (or bare nuclei). The same rule applies to the injector itself: a higher charge state of ions produced in the source will result in a more efficient acceleration and a smaller, simpler and less costly injector. The design of the Chiba facility illustrates this point: the beam from the ion source is accelerated in a $7.3 \mathrm{~m}$ long RFQ, followed by a $24 \mathrm{~m}$ long linear accelerator, all in order to give partially stripped ions enough energy for an efficient full stripping at injection into the synchrotron. The second, also important consideration are losses of ions in collisions with molecules of the residual gas in the vacuum chamber; they will be lowest for fully stripped ions.

\section{A) Low Charge State Ion Sources}

A typical representative of low charge state ion sources and also one still frequently used in accelerators is the Penning or PIG (from Philips Ionization Gauge) ion source. It is very simple in principle, consisting of two cathodes placed at each end of a hollow, cylindrical anode; the electrode structure is immersed in a magnetic field. Electrons emitted from either cathode are accelerated by the cathode electric field into the hollow anode where they are trapped and forced to make many oscillations along the magnetic field lines before they are lost to the anode. Electrons with a sufficient energy will ionize particles in the source volume and a plasma will be created there. Penning sources are capable of producing copious ion currents of any element, but in a rather low ionization state and with a broad charge state distribution; the reason for the former is a relatively low cathode to anode voltage that the plasma in the source can sustain.

The standard approach in the design of a high energy ion accelerator (such as for cancer treatment), based on a low charge state ion source, is to select a charge state with a sufficient intensity, then preaccelerate the ions to an intermediate level and pass them through a thin stripping target. At the exit of the stripper charge states of ions will be higher than before but their spectrum will be broader; the price to pay is the fact that unwanted states will have to be rejected, resulting in a substantial loss of intensity. In some cases the process has to be repeated once more, by further acceleration and final stripping before injection into the synchrotron, as it was proposed for the first TERA project. It is evident that this scheme, although based on a simple inexpensive ion source, could be more costly concerning the overall acceleration process itself.

B) Intermediate Charge State Ion Sources - ECR

An Electron Cyclotron Resonance (ECR) ion source is again a magnetic electron trap, where a plasma is created and maintained in a minimum B-field. Ionization is performed by fast electrons in a step-by-step process, resulting in an increase of the average charge state of ions. Electrons are heated by high frequency electromagnetic waves introduced into the plasma; there is a region in the plasma where the wave frequency is in resonance with the magnetic field. ECR ion sources have 
been in a wide use for several decades, they are reliable and easy to operate, although much more expensive than e.g. a Penning source. They are capable of producing intermediate charge states of many elements, up to uranium, but their performance with light ions is what is relevant for medical applications. The best yields of carbon or oxygen ions of modern ECR ion sources is in the heliumlike state, with two electrons remaining, while for heavier ions the optimum charge state is lower. Still, an ECR ion source for use in a medical accelerator would require just one stripping stage to produce bare nuclei, with the final yield not much different from a Penning source. This type of ion sources is being used or proposed as an alternative to Penning sources, e.g. at Chiba or in the TERA proposal. The work on improving ECR sources is going on at many laboratories, but it is doubtfull that in a foreseeable future encugh progress will be made to produce a high enough yield of fully stripped light ions.

C) High Charge State Ion Siources - EBIS

An Electron Beam Ion Source (EBIS) is a device where electrons and ions are confined in a combination of electrostatic and magnetic fields. The magnetic field is solenoidal, serving to compress and confine a high current density electron beam. The negative space charge of electrons confines the ions radially while a system of coaxial electrodes confines them axially via properly selected potentials. The process of ionization is again step-by-step, in collisions between fast electrons in the beam and confined ions. An EBIS is in principle a pulsed device, where the process starts with the injection of neutral particles or very low charge state ions of the desired species. During the confinement time, which can be chosen at will, the charge state distribution of confined ions moves from lower to higher values; the final distribution depends on the electron beam current density and the confinement time. These two parameters can be easily adjusted so that the source is able to produce any ion specie; in any charge state (e.g., fully stripped uranium). For light ions up to neon satisfactory yields of bare nuclei have already been achieved, while for heavier ions such as argon yields are still too low. The comparison of performances of an ECR source with an EBIS is not straightforward: an ECR source is in principle a device delivering a certain current, while an EBIS delivers a certain positive charge depending on the electron beam parameters and size of the device. Thus the ion current from an EBIS will depend also on the selected value of the ion pulse length, which is adjustable within a certain range. The latter property of an EBIS makes this source very suitable for injection into a synchrotron because a very high current can be injected into the ring during a short interval. At present the work on EBIS development is proceding at several laboratories and within a few years a simple, room temperature device should become available delivering the ion intensities needed for medical synchrotrons. The main advantage of an EBIS is its possibility of producing fully stripped ions, up to neon with a sufficient intensity, eliminating thus the need for any stripping before injection into the synchrotron and making the injector short, simple and less expensive.

Injector

The simplest design of an injector results if the source can deliver fully stripped ions. At present it is only an EBIS that is capable of delivering sufficient intensities of fully stripped ions, but should an ECR be developed in the future with the similar performance, the choice will be between these two types, with other characteristics deciding which one to use. In any case, the only accelerator stage between the source and the synchrotron ring will be just a short RFQ, with an 
energy high enough to accelerate and inject the required number of ions. Elimination of stripping foils will make the design simpler, more reliable and easier to operate.

\section{Beam Delivery System}

The only beam delivery system presently in use on proton and light ion accelerators for tumor treatment is of the passive type. The active type systems, with accelerator energy modulation and beam raster or voxel scanning, will be tested in the near future at the GSI facility. Although active systems seem to be more complex, once the required technology is developed (accelerator controls, beam intensity monitoring and controls, interface between the beam delivery system and patient) the most important element will be to determine the exact location, shape and properties of the tumor, as well as properties of tissues ahead of the tumor. The knowledge of the properties of healthy tissues is of crucial importance not only because they will determine what will happen to the beam when passing toward the tumor but because it will serve to estimate the dose delivered to healthy organs. However, the design of the accelerator should be such as to satisfy the requirements of either a passive or an active beam delivery system.

\section{COST EFFECTIVENESS OF LIGHT ION CANCER THERAPY}

There are two issues to consider when estimating the cost effectiveness of light ion cancer therapy: costs of treatment of a patient as compared with other modalities having similar prospects for cure of cancer and costs of treatment of a patient for whom there are no other (or better) modalities for cure compared to costs of other life-saving procedures. While the first issue is the question of economics - how to find the most cost-effective treatment if several modalities with similar outcomes are available, the second issue borders with the question how to determine whose life should be saved.

One of the most thorough analyses of costs of a proton/light ion facility has been done for the TERA project [3]; estimates are available for a few other projects as well [9][10][17]. The Phase I of this project would be limited to production and use of protons only, while the addition of light ions up to oxygen has been considered as an upgrade. The total cost of the facility has been estimated at about \$M 50, with the light ion upgrade adding about \$M 7.5; this includes management, installation and commissioning costs and $15 \%$ contingency. It is interesting to note that the accelerator itself contributes less than $20 \%$ to the total construction cost of the facility. To estimate the cost of the treatment per patient several assumptions had to be made in the proposal; the first was about the number of patients that could be treated per year. After an initial period of two years, and operating in two shifts, about 1000 patients could be treated per year ( this number could be increased by adding more treatment rooms or by operating in three shifts which is a standard mode of operation of nuclear or high energy physics machines). Assuming a 25 year amortization and including the operating costs, the estimated cost per patient was about $\$ 15000$ for proton therapy; light ion therapy would be more expensive by about $20 \%$. A somewhat lower estimate has been arrived at for a German project. If, instead, 1500 patients are treated per year, the cost would be reduced correspondingly. There are two major proton therapy facilities in the United States, Loma Linda and Harvard Cyclotron Laboratory; their charges have been quoted in a wide range, from 
$\$ 10000$ to $\$ 60000$, depending on the number of sessions. At present there are no light ion facilities either existing or proposed to he built in the United States.

It is not easy to compare these costs with other modalities for cancer treatment, again because of a broad range of costs, from one country to another and depending on the extent of the disease. In Germany the average cost of conventional radiotherapy amounts to about $\$ 5000$, but the modern conformal radiation treatment would be more expensive; oncological surgery is more expensive, average about $\$ 10000$ and chemotherapy even more, up to $\$ 40000$. In the USA the structure of costs is different, so that e.g. an oncological surgery may easily cost $\$ 25000$. It would follow from these data that a proton therapy treatment is about twice as expensive as conventional radiotherapy, while the light ion treatment rnay be up to three times more expensive. Average costs of proton therapy are comparable to oncological surgery, but lower than for chemotherapy. However, there are other factors to be taken into account, such as the length of the stay in the hospital (which in some cases is the major contribution to the total cost), overall treatment time, quality of life and socioeconomic disruption of life, and acute and long term morbidity. When all these factors are included it may well be that a somewhat more expensive modality, such as radiotherapy with protons or even with light ions, would still be preferable.

Finally, addressing the second issue, one should consider costs of other socially and economically acceptable treatments, such as bone marrow transplantation which may cost up to $\$ 100000$, and heart transplantation which may cost up to $\$ 140000$ for the surgery alone and several hundred thousand with hospital and drug costs included. The latter procedure, long term lifesaving in about $60 \%$ of cases, is presently limited not by the costs of the treatment itself but by the number of available organs. However, this issue is beyond the scope of this report.

\section{CONCLUDING REMARKS}

Light ions have several distinct characteristics that seem to offer a more promising treatment of some types of cancer than other types of radiation. Their physical selectivity in the dose delivery is very good, with lower scattering and enhanced energy deposition at the end of the track. Radiobiological properties, sich as a reduced sensitivity to the phase in the cell cycle, a lower oxygen enhancement ratio and higher values of factors LET and RBE also seem attractive and advantageous for cancer treatment. Analyses performed for several projects have shown that there are a number of types of cancer where light ions may offer a much better or the only prospect for cure and, therefore, cannot be considered to compete with established methods but to complement them. Still, the number of patients treated so far has been relatively very small and limited to just two facilities, Berkeley and Chiba. 'There are several reasons for the reluctance to introduce this new type of radiation as a modality in ciancer treatment and we shall try to address them.

It is a fact that except for the dedicated Chiba facility all other accelerators capable of producing energetic light ions were built for a different purpose and with different characteristics than needed for the therapy. The range of their parameters (ion species, energy, intensity) is usually much broader and the construction and operating costs much higher than acceptable for medical applications; the facilities tend to be complex and not reliable enough. Because tumor treatment is not their primary purpose, the time available for radiobiological studies and trials is limited. The Chiba facility, once in full operation, will be able to supply the needed ion beams and in a few years valuable experience will be gained about the effectiveness and advantages of light ions. The new 
Hyogo facility should be in operation by the year 2001 and add more data to the statistics. With these two facilities Japan has taken a leading role in exploring the merits of light ions. European efforts, although very important for the progress of the field, are concentrated on limited clinical studies at the GSI and on TERA Project and Med-AUSTRON initiative. If these efforts result in the construction of a dedicated light ion facility in Europe, this will be again an important step in determining the feasibility of light ion use in cancer treatment. In the United States, unfortunately, at present there is no ongoing effort, after the closing of the Bevalac facility. The only accelerator capable of producing light ions for cancer treatment is the Booster at Brookhaven National Laboratory but presently there are no plans to use its beams in medicine although it is likely to be used for radiobiological research.

Characteristics of light ions that make them attractive for cancer treatment, are as well the reason that their application is much more critical and complex. Tumor diagnostics and beam delivery systems become very sophisticated, and so do the accelerator controls. However, after the initial investment into the development of the hardware and software of a prototype facility, the next generation should become simple enough to be operated in a hospital environment. Results from GSI studies in accelerator control and active beam delivery systems will be very important for further developments.

Progress in the use of conventional types of radiation, including protons, has been substantial and the beam delivery has moved closer to the ideal, conform treatment. This has been mentioned as an argument against the introduction of new types of radiation in tumor treatment. However, light ions were never supposed to replace the methods which have already achieved excellent results but to try to treat those cases for which other modalities offer little or no hope. To prove or disprove the expectations, based on physical and radiobiological properties, it is necessary to broaden the studies and clinical trials to get better statistics.

Finally, there is the question of cost-effectiveness compared to other modalities. Again, it may be true that conventional radiation treatments are less costly than one with light ions, but here the argument is the same as before: if light ion treatment is a much better or the only modality available for certain tumor sites then the cost should be of secondary importance, considering other more expensive, but life saving procedures.

To conclude, for such a universal medical problem as cancer it is important to explore all avenues to accomplish a cure. Radiation with light ions offers a possibility to improve existing methods but the number of patients is still far too small to reach the judgement about its benefits and advantages. In the present situation one cannot expect that funds would become available to start construction of new facilities above those mentioned before. It is therefore important that the facilities already existing, including those built for other purpose, are fully utilized for radiobiological studies and clinical trials of a limited number of tumor sites where other methods fail or are not very successful; an international cooperation will be indispensable to achieve the desired results. 


\section{ACKNOWLEDGEMENT}

This work was performed under the auspices of the U. S. Dept. of Energy. The generosity of the Rockefeller Foundation in awarding me the residency at the Bellagio Study and Conference Center to write the report itself, is greatly appreciated. Professor U. Amaldi kindly gave the permission to use figures from Ref. 2. Discussions with and advice received from Dr. J. Sisterson, Harvard Cyclotron Laboratory, are also acknowledged.

\section{REFERENCES}

1. Ion Beams in Tumor Therapy, U. Linz, ed., Chapman \& Hall (1995)

2. The TERA Project and the Centre for Oncological Hadrontherapy, INFN-LNF-Divisione Ricerca, Frascati (Italy), U. Amaldi and M.Silari, eds, July 1994

3. The RITA Network and the Design of Compact Proton Accelerators, INFN-LNF- Divisione Ricerca, Frascati (Italy), U. Amaldi, M. Grandolfo and L. Picardi, eds, August 1996

4. Proc. First Int. Symp. Hadrontherapy, Como (Italy), October 1993, U. Amaldi and B. Larsson, eds, Elsevier (1994)

5. Proc. Third European Particle Accelerator Conference EPAC 92, Editions Frontieres (1992)

6. Proc. Fourth European Particle Accelerator Conference EPAC 94, World Scientific Publishing Co. (1994)

7. Proc. Fifth European Particle Accelerator Conference EPAC 96, Institute of Physics Publishing (1996)

8. Proc. NIRS Int. Seminar on Appl. of Heavy Ion Accelerator to Radiation Therapy of Cancer, T. Kanai and E. Takada, eds, Nat. Inst. of Radiological Sciences, Japan (1994)

9. E. Pedroni, in Ref. 6, p.407

10. L.H. Schwartz, et al., Bull.Cancer/Radiotherapie, Vol.82, p.365 (1995)

11. U. Linz, in Ref. 1, p.15

12. D.E. Bonnett, Phys.Med.Biol. Vol.38, p.1371 (1993)

13. A. Wambersie, in Hadrons in Radiation Therapy, Proc. European Heavy Particle Therapy Group Meeting, Orleans, May 1995; Bul. du Cancer/Radiotherapy, Vol.83, p.68 (1996) 
14. K. Ando, et al., in Ref. 8, p.32

15. J. Alonso, in Proc. 1995 Particle Accelerator Conference, IEEE Service Center, p.58

16. U. Amaldi, in Proc. XVIII Intern. Linear Accelerator Conf., Geneva 1996, CERN (1996), p.605

17. Proton Therapy Facility at BNL, Report to the BNL Director, March 1995 (unpublished)

18. G. Gademann, in Ref. 4 , p.59

19. Particles, a Newsletter, Proton Therapy Co-Operative Group, J. Sisterson, ed.

20. J.E. Munzenrider, in Ref. 1, p.95

21. A. Smith, et al., in Ref. 8, p.50

22. Y. Jongen, in Ref. 8 , p.59

23. P. Chauvel, Radiat.Environ.Biophys., Vol.34, (1995), p.49

24. J.M. Cosset, et al., Radiat.Environ.Biophys., Vol.34 (1995), p.3739

25. J.E. Munzerider, in Ref. 4, p.83

26. A. Smith, in Ref. 4 , p.138

27. J.M. Slater, in Ref. 4, p. 130

28. E. Pedroni, in Ref. 8, p.259

29. H. Blattmann, et al., in Ref. 4, p.122

30. E. Egger and L. Zografos, in Ref. 4, p.145

31. H. Tsujii, et al., in Ref. 1, p.127

32. E.I. Minakova, in Ref.1, p.106

33. E.I. Minakova, in Ref. 4, p.102

34. J.R. Castro, Radiat.Environ.Biophys., Vol.34 (1995), p.45

35. J.R. Castro, Int.J.Rad.Onc.Biol.Phys., Vol.29 (1994), p.647

36. E.A. Blakely and J.R. Castro, in Ref. 8, p.149 
37. P.K. Lillis-Hearne and J.R.. Castro, in Ref. 1, p.133

38. R.P. Levy, et al., in Ref. 1, p.142

39. J.R. Castro, in Ref. 4, p.209

40. H. Tsujii, et al., in Ref. 8, p.212

41. H. Tsujii and Y. Hirao, 6th China-Japan Joint Symp.on Accelerators for Nuclear Science and their Applications, Chengdu (China), (1996)

42. K. Morita, et al., in Ref.7, p.237

43. K. Kawachi, et al., in Ref. 4, p.229

44. H. Eickhoff, et.al., in Ref. 7, p.2641

45. G. Kraft, et al., in Ref. 4, p. 217

46. J.M. Slater, et al., in Ref. 1, p.319

47. G. Coutrakon, et al., in Ref. 4, p.282

48. K. Kawachi, in Ref. 8, p.10

49. S. Yamada, et al., in Ref. B, p.13

50. K. Ueda, et al., in Ref. 8, p..19

51. T. Murakami, et al., in Proc. XVIII Int. Linear Accelerator Conference, Geneva 1996, CERN (1996), p.830

52. E. Takada, et al., in Ref. 7, p.2659

53. G. Kraft, in Ref. 1, p.341

54. K. Blasche and B. Franczik, in Ref. 5, p.9

55. B. Franczak, in Ref. 7, p.2647

56. U. Linz and R. Maier, in Ref. 5, p.1672

57. U. Linz, in Ref. 4, p.386

58. U. Amaldi, in Ref. 4, p.45 
59. A. Itano, in Ref. 8, p. 88

60. J.R. Alonso, in Ref. 1, p.171

61. P. Mandrillon, in Ref. 1, p.181 